\title{
Pacific
}

Journal of

Mathematics

\section{PROPERNESS, CAUCHY INDIVISIBILITY \\ AND THE WEIL COMPLETION \\ OF A GROUP OF ISOMETRIES}

Antonios Manoussos And POLYChronis StRantzalos

Volume $259 \quad$ No. 2

October 2012 


\title{
PROPERNESS, CAUCHY INDIVISIBILITY AND THE WEIL COMPLETION OF A GROUP OF ISOMETRIES
}

\author{
Antonios Manoussos And Polychronis StRAntzalos
}

\begin{abstract}
Investigating the impact of local compactness and connectedness in the theory of proper actions on locally compact and connected spaces, we introduce a new class of isometric actions on separable metric spaces called Cauchyindivisible actions. The new class coincides with that of proper actions on locally compact metric spaces, without assuming connectivity, and, as examples show, may be different in general. In order to provide some basic theory for this new class of actions, we embed a Cauchy-indivisible action in a proper action of a semigroup in the completion of the underlying space. We show that, if this semigroup is a group, there are remarkable connections between Cauchy indivisibility and properness, while the original group has a Weil completion and vice versa. Further connections in this direction establish a relation between Borel sections for Cauchy-indivisible actions and fundamental sets for proper actions. Some open questions are added.
\end{abstract}

\section{Introduction}

In the paper at hand, having in mind the fruitful theory of proper transformation groups on locally compact and connected spaces, we propose an analogous class of actions, not necessarily proper, without assuming local compactness and connectedness of the underlying spaces. So, we introduce a new, rather natural, class of metric actions on separable (not necessarily connected) metric spaces called Cauchy-indivisible. Note that isometric actions constitute nowadays an important part of the theory of proper actions and that the group of isometries of a locally compact and connected metric space acts properly on it.

As the following definition shows, Cauchy-indivisible actions are characterized by an isotropic behavior of divergent nets of the acting group with respect to the basic metric notion of a Cauchy sequence. Recall that $z_{i} \rightarrow \infty$ in $Z$ means that the net $\left\{z_{i}\right\}$ does not have any convergent subnet in the space $Z$.

During this research the first author was fully supported by SFB 701 "Spektrale Strukturen und Topologische Methoden in der Mathematik" at the University of Bielefeld, Germany.

MSC2010: primary 37B05, 54H20; secondary 54H15.

Keywords: proper action, Weil completion, Cauchy indivisibility, Borel section, fundamental set. 
Definition 1.1. Let $(G, X)$ be a continuous action of a topological group $G$ on a metric space $X$. The action is said to be Cauchy-indivisible if the following holds: If $\left\{g_{i}\right\}$ is a net in $G$ such that $g_{i} \rightarrow \infty$ in $G$ and $\left\{g_{i} x\right\}$ is a Cauchy net in $X$ for some $x \in X$ then $\left\{g_{i} x\right\}$ is a Cauchy net for every $x \in X$.

It turns out that a Cauchy-indivisible action on a locally compact or complete metric space is proper and vice versa (see Section 3), and that in general the two notions may differ (see Section 4). In both cases the underlying space is not assumed to be connected. The omission of this assumption in the locally compact case, as well the omission of local compactness in the main part of the paper at hand is an advantage coming from the fact that Cauchy indivisibility essentially reflects the global character of self-maps of $X$ compared with the local properties or the connectedness of the underlying space. So we can generalize the framework of proper actions and go beyond, provided that this new framework leads

(a) to interesting results in the non locally compact case, and

(b) enables a better understanding of proper actions on locally compact spaces.

Concerning requirement (b) we note that in Theorem 3.3 we give an answer to the open question of characterizing proper actions on nonconnected locally compact metric spaces and in Theorem 7.4 we establish an interconnection between Borel sections (which occur in Cauchy-indivisible actions on separable spaces, see Proposition 7.1) and fundamental sets that characterize proper isometric actions. Recall that a section of an action $(G, X)$ is a subset of $X$ which contains only one point from each orbit. A Borel section is a section that is a Borel subset of $X$ (useful, for example, in measure theory).

Theorem 7.4. Let $G$ be a group which acts properly on a locally compact space $X$, and suppose that the orbit space $G \backslash X$ is paracompact. Let $S$ be a section for the action $(G, X)$.

(i) For every open neighborhood $U$ of $S$ we can construct a closed fundamental set $F_{c}$ and an open fundamental set $F_{o}$ such that $F_{c} \subset F_{o} \subset U$.

(ii) If, in addition, $(X, d)$ is a separable metric space, in which case the action $(G, X)$ is Cauchy-indivisible, then there exists a Borel section $S_{B}$, which is also a fundamental set, such that $S_{B} \subset F_{c} \subset F_{o} \subset U$.

Note that $S_{B}$ in (ii) of the above theorem is a "minimal" fundamental set, because of its construction, and as such may lead to applications.

The new notion of "like properness" seems to be suitable for structure theorems, as our first results indicate. Concerning requirement (a) above, in Section 5, which is the main part of the paper at hand, we consider a separable metric space $(X, d)$ such that the natural evaluation action of the group of isometries $\operatorname{Iso}(X)$ on $X$ is Cauchy-indivisible. Let $\widehat{X}$ denote the completion of $(X, d)$ and let $E$ be the Ellis 
semigroup of the lifted group $\widehat{\operatorname{Iso}(X)}$ in $C(\widehat{X}, \widehat{X})$, that is, the pointwise closure of $\widehat{\operatorname{Iso}(X)}$ in $C(\widehat{X}, \widehat{X})$. Let

$$
\begin{aligned}
H & =\left\{h \in C(\widehat{X}, \widehat{X}) \mid \text { there exists a sequence }\left\{g_{n}\right\} \subset \operatorname{Iso}(X)\right. \\
\left.\quad \text { with } g_{n} \rightarrow \infty \text { in } \operatorname{Iso}(X) \text { and } \hat{g}_{n} \rightarrow h \text { in } C(\widehat{X}, \widehat{X})\right\}, & \\
X_{l}=\{h x \mid h \in H, x \in X\}, & \\
X_{p} & =\{h x \mid h \in H \cap \operatorname{Iso}(\widehat{X}), x \in X\} .
\end{aligned}
$$

With this notation and the previously mentioned assumptions, among other results we show the following.

Theorem 5.13. The set $X \cup X_{p}$ is the maximal subset of $X \cup X_{l}$ that contains $X$ such that the map

$$
\omega: E \times\left(X \cup X_{p}\right) \rightarrow\left(X \cup X_{p}\right) \times \widehat{X},
$$

with $\omega(f, y)=(y, f y), f \in E$, and where $y \in X \cup X_{p}$ is proper.

The interest in this theorem lies in the fact that an action $(G, X)$ is proper if the map $G \times X \rightarrow X \times X$ defined by $(g, x) \mapsto(x, g x)$ is proper, see [Bourbaki 1966a, Definition 1, p. 250].

We recall that a topological group has a Weil completion with respect to the uniformity of pointwise convergence if it can be embedded densely in a complete group with respect to its left uniform structure.

Proposition 5.18. The following are equivalent:

(i) The map $\omega: E \times\left(X \cup X_{l}\right) \rightarrow\left(X \cup X_{l}\right) \times \widehat{X}$ is proper.

(ii) $E$ is a group (precisely a closed subgroup of $\operatorname{Iso}(\widehat{X})$ ).

(iii) Iso(X) has a Weil completion.

Corollary 5.20. If $E$ is a group the action (Iso $(X), X)$ is embedded densely in the proper action $\left(E, X \cup X_{l}\right)$ such that the following equivariant diagram commutes:

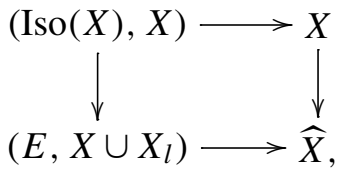

where $X \rightarrow X \cup X_{l}$ is the inclusion map and the map $\operatorname{Iso}(X) \rightarrow E$ is defined by $g \mapsto \hat{g}$ for every $g \in \operatorname{Iso}(X)$. By "densely" we mean that $X$ is dense in $X \cup X_{l}$ and $\widehat{\operatorname{Iso}(X)}$ is dense in $E$. 
The above result may lead to further structure theorems, see Question 5.21.

Proposition 7.1. If the Ellis semigroup $E$ is a group then the action $\left(E, X \cup X_{l}\right)$ has a Borel section.

As Theorem 7.4(ii) mentioned above indicates, the notion of a Borel section, which according to the above result is a feature of the Cauchy-indivisible actions on separable metric spaces, is remarkably related to that of a fundamental set in the locally compact case and may be, similarly, used for structural theorems. So, it is interesting to ask whether the existing Borel section for the action $\left(E, X \cup X_{l}\right)$ can be reduced to a Borel section for the initial action (Iso( $X), X)$, see Question 7.7.

In order to indicate or to exclude possible directions for further investigation concerning Cauchy-indivisible actions, we study various examples, see Examples 5.14, 5.17, 5.23, and 7.3. Among them, an example that may be of independent interest is the following (see Section 6): Consider the action (Iso(Iso( $\mathbb{Z}))$, Iso( $\mathbb{Z})$ ), where $\mathbb{Z}$ is the discrete space of the integers, with suitable metrics on the acting group $\operatorname{Iso}(\operatorname{Iso}(\mathbb{Z}))$ and the underlying space $\operatorname{Iso}(\mathbb{Z})$. We show that this action is proper and Cauchy-indivisible while the Ellis semigroup is not a group and Iso(Iso(ZZZ)) has no Weil completion.

\section{Basic notions and notation}

For what follows, in addition to the notation established in the introduction, $(X, d)$ will denote a metric space with metric $d$ and $\operatorname{Iso}(X)$ will denote its group of (surjective) isometries of $X$ endowed with the topology of pointwise convergence. With this topology Iso $(X)$ is a topological group [Bourbaki 1966b, Chapter $X, \S 3.5$, Corollary]. Let $(\widehat{X}, \hat{d})$ stand for the completion of $(X, d)$. For a Cauchy sequence $\left\{x_{n}\right\}$ in $X$ let $\left[x_{n}\right] \in \widehat{X}$ denote the limit point of $\left\{x_{n}\right\}$ in $\widehat{X}$. We denote by $\hat{g}$ and Iso( $(X)$ the lift of $g \in \operatorname{Iso}(X)$ and the lift of the group Iso( $X)$, respectively, in $C(\widehat{X}, \widehat{X})$, the space of the continuous self-maps of $\widehat{X}$ (which is considered with the topology of pointwise convergence).

A continuous action of a topological group $G$ on a topological space $X$ is a continuous map $G \times X \rightarrow X$ with $(g, x) \mapsto g x, g \in G, x \in X$ such that $(e, g) \mapsto x$, for every $x \in X$ where $e$ denotes the unit element of $G$, and $(h,(g, x)) \mapsto(h g) x$ for every $h, g \in G$, and $x \in X$. When the action map is known we will denote the action simply by $(G, X)$. Let $U \subset X$, then $G U:=\{g x \mid g \in G, x \in U\}$. Especially, if $U=\{x\}$ then the set $G x:=G\{x\}$ is called the orbit of $x \in X$ under $G$. The subgroup $G_{x}:=\{g \in G \mid g x=x\}$ of $G$ is called the isotropy group of $x \in X$. The natural evaluation action of $\operatorname{Iso}(X)$ on $X$ (denoted by (Iso $(X), X)$ ) is the map Iso $(X) \times X \rightarrow X$ with $(g, x) \mapsto g(x), g \in \operatorname{Iso}(X)$, and $x \in X$. If we endow $\operatorname{Iso}(X)$ with the topology of pointwise convergence this action is always continuous. As usual, $S(x, \varepsilon)$ will denote the open ball centered at $x$ with radius $\varepsilon>0$. 
Definition 2.1. A continuous action $(G, X)$ is (equivalently to the Bourbaki definition) proper if $J(x)=\varnothing$, for every $x \in X$, where

$$
\begin{aligned}
& J(x)=\left\{y \in X \mid \text { there exist nets }\left\{x_{i}\right\} \text { in } X, \text { and }\left\{g_{i}\right\} \text { in } G\right. \\
& \text { with } \left.g_{i} \rightarrow \infty, \lim x_{i}=x \text { and } \lim g_{i} x_{i}=y\right\}
\end{aligned}
$$

denotes the extended (prolongational) limit set of $x \in X$.

It is easily seen that in the special case of actions by isometries $J(x)=L(x)$ holds for every $x \in X$, where

$$
L(x)=\left\{y \in X \mid \text { there exists a net }\left\{g_{i}\right\} \text { in } G \text { with } g_{i} \rightarrow \infty \text { and } \lim g_{i} x=y\right\}
$$

denotes the limit set of $x \in X$ under the action of $G$ on $X$. Hence an action by isometries $(G, X)$ is proper if and only if $L(x)=\varnothing$ for every $x \in X$.

\section{Cauchy indivisibility and proper actions on locally compact metric spaces}

In this section we show that for group actions on locally compact metric spaces the notions of properness and Cauchy indivisibility coincide. We start with the following easily proved observation.

Lemma 3.1. Let $(X, d)$ be a locally compact metric space and $\left\{g_{i}\right\} \subset \operatorname{Iso}(X)$ be a net such that $\left\{g_{i} x\right\}$ is a Cauchy net for some $x \in X$. Then there exists a point $y \in X$ such that $g_{i} x \rightarrow y$.

Proposition 3.2. Let $(X, d)$ be a locally compact metric space. The action (Iso( $X)$, $X)$ is proper if and only if it is Cauchy-indivisible.

Proof. Assume that (Iso $(X), X)$ is Cauchy-indivisible. We will show that the limit sets $L(x)$ are empty for every $x \in X$. Assume the contrary, that is, there exist a net $\left\{g_{i}\right\}$ in $\operatorname{Iso}(X)$ and $x, y \in X$ such that $g_{i} \rightarrow \infty$ and $g_{i} x \rightarrow y$. We will show that $g_{i} \rightarrow h$ for some $h \in \operatorname{Iso}(X)$, which is a contradiction of the assumption $g_{i} \rightarrow \infty$. Since ( $\operatorname{Iso}(X), X)$ is Cauchy-indivisible then $\left\{g_{i} x\right\}$ is a Cauchy net, for every $x \in X$. Therefore, by the previous lemma, there is a map $h: X \rightarrow X$ defined by $h(x):=y$ such that $g_{i} \rightarrow h$ pointwise on $X$ and $h$ preserves the metric $d$. Observe that $g_{i}^{-1} y \rightarrow x$ since $d\left(g_{i}^{-1} y, x\right)=d\left(y, g_{i} x\right)$. Applying Cauchy indivisibility for the action (Iso $(X), X)$ and the previous lemma again, we conclude that there exists a map $f: X \rightarrow X$ such that $g_{i}^{-1} \rightarrow f$ pointwise on $X$ and $f$ preserves the metric $d$. Obviously $f$ is the inverse map of $h$, hence $h \in \operatorname{Iso}(X)$.

The converse implication follows easily in a similar way.

If $X$ is locally compact and $G$ acts properly on $X$ (hence $G$ is a locally compact group), it is well known, see, for example, [Koszul 1965], that there exists a $G$-invariant compatible metric on $X$. Compatible means that this metric induces 
the topology of $X$. Hence, the previous proposition states the following result that characterizes the properness of actions on locally compact metric spaces independently of the connectedness of the underlying space.

Theorem 3.3. Let $(X, d)$ be a locally compact metric space. An action $(G, X)$ is proper if and only if it is Cauchy-indivisible.

Remark 3.4. The previous theorem also holds, and can be similarly proved, if we replace the full group of isometries of $X$ by a closed subgroup of it or if we replace the local compactness of $X$ by completeness.

\section{Cauchy indivisibility vs. properness}

In this section we provide examples showing that Cauchy indivisibility and properness are distinct notions for isometric actions on separable and non locally compact metric spaces. We also provide some criteria for the coexistence of Cauchyindivisible and proper actions on the basis of the dynamical behavior of the lifting of the action ( $\operatorname{Iso}(X), X)$ in the completion of the underlying space.

Remark 4.1. The example in Section 6 shows that the two notions may coexist also in the case when $X$ is neither locally compact nor complete.

The following example shows that the action (Iso $(X), X)$ can be proper and not Cauchy-indivisible.

Example 4.2. Let $X$ be the set $\mathbb{Q}$ of the rational numbers endowed with the Euclidean metric. It is easy to see that the action $(\operatorname{Iso}(X), X)$ is proper. Take a sequence of rational numbers $\left\{q_{n}\right\}$ such that $q_{n} \rightarrow a$, where $a$ is an irrational. Let $\left\{g_{n}\right\} \subset \operatorname{Iso}(X)$ with $g_{n} x:=(-1)^{n} x+q_{n}$ for every $x \in X$, then $g_{n} \rightarrow \infty$ in $\operatorname{Iso}(X)$. Since $g_{n} 0=q_{n}$ for every $n \in \mathbb{N}$, the sequence $\left\{g_{n} 0\right\}$ is Cauchy. But for $x \neq 0$ the sequence $\left\{g_{n} x\right\}$ has two limit points in $\mathbb{R}$ and hence cannot be a Cauchy sequence.

The next example shows that the action (Iso $(X), X)$ can be Cauchy-indivisible and not proper.

Example 4.3. Let $X$ be the set $\mathbb{Q}+\sqrt{2} \mathbb{N}$ endowed with the Euclidean metric. Its group of isometries is $\mathbb{Q}$ acting by translations (reflections are excluded because of the addend $\sqrt{2} \mathbb{N}$ ). Therefore, (Iso $(X), X)$ is Cauchy-indivisible. However, the action $(\operatorname{Iso}(X), X)$ is not proper. To see that take a sequence of rational numbers $\left\{q_{n}\right\}$ such that $q_{n} \rightarrow \sqrt{2}$. Let $\left\{g_{n}\right\} \subset \operatorname{Iso}(X)$ with $g_{n} x:=x+q_{n}$. Observe that $g_{n}^{-1} \sqrt{2} \rightarrow 0 \notin X$. Therefore $g_{n} \rightarrow \infty$ in Iso $(X)$. Since $g_{n} \sqrt{2} \rightarrow 2 \sqrt{2} \in X$ the limit set $L(\sqrt{2})$ is not empty, so the action (Iso $(X), X)$ is not proper.

Motivated by these examples we give necessary and sufficient conditions for a Cauchy-indivisible action (Iso $(X), X)$ to be proper and vice versa:

Proposition 4.4. Let Iso $(X)$ be Cauchy-indivisible. The following are equivalent: 
(i) The action (Iso $(X), X)$ is proper.

(ii) If $h$ is in the pointwise closure of $\widehat{\operatorname{Iso}(X)}$ in $C(\widehat{X}, \widehat{X})$ then either $h(X) \subset X$ or $h(X) \subset \widehat{X} \backslash X$.

Proof. Assume that the action $(\operatorname{Iso}(X), X)$ is proper and $h$ is in the pointwise closure of $\widehat{\operatorname{Iso}(X)}$ in $C(\widehat{X}, \widehat{X})$. Then there is a net $\left\{\hat{g}_{i}\right\}$ in $\widehat{\operatorname{Iso}(X)}$ such that $\hat{g}_{i} \rightarrow h$ pointwise in $\widehat{X}$. If $h(X) \cap X \neq \varnothing$ then there is some $x \in X$ such that $\hat{g}_{i} x \rightarrow h x \in$ $X$. Since the action (Iso $(X), X)$ is proper the net $\left\{g_{i}\right\}$ has a convergent subnet in $\operatorname{Iso}(X)$. Then it is easy to see that $h \in \widehat{\operatorname{Iso}(X)}$, hence $h(X) \subset X$.

Assume now that condition (ii) holds. We will show that the limit sets $L(x)$ are empty for every $x \in X$, hence the action (Iso $(X), X$ ) is proper. We will proceed by contradiction. Assume that there exist $x, y \in X$ and a net $\left\{g_{i}\right\}$ in $\operatorname{Iso}(X)$ with $g_{i} x \rightarrow y$ and $g_{i} \rightarrow \infty$ in $\operatorname{Iso}(X)$. Since $\left\{g_{i} x\right\}$ is a Cauchy net in $X$ and $\operatorname{Iso}(X)$ is Cauchy-indivisible then $\left\{g_{i} x\right\}$ is a Cauchy net for every $x \in X$, hence $\left\{g_{i} x\right\}$ converges in $\widehat{X}$ for every $x \in X$. So, we can define a map $h: X \rightarrow \widehat{X}$ by letting $h x:=\lim \hat{g}_{i} x$. It is easy to see that $h$ preserves the metric $\hat{d}$ on $X$. Thus, if $w \in \widehat{X}$ and $\left\{x_{n}\right\} \subset X$ is a sequence in $X$ such that $x_{n} \rightarrow w$ in $\widehat{X}$ then $\left\{h x_{n}\right\}$ is a Cauchy sequence in $X$, hence it converges to a point in $\widehat{X}$ which is independent of the choice of the sequence $\left\{x_{n}\right\}$. Then, by [Bourbaki 1966a, Chapter I, $\S 8.5$, Theorem 1], the map $h: X \rightarrow \widehat{X}$ has a unique continuous extension on $\widehat{X}$. It is easy to see that $\hat{g}_{i} \rightarrow h$ pointwise on $\widehat{X}$, thus $h$ is in the pointwise closure of $\widehat{\operatorname{Iso}(X)}$ in $C(\widehat{X}, \widehat{X})$. Since $g_{i} x \rightarrow y$ then $h x=y$ where $x, y \in X$. So using our hypothesis $h(X) \subset X$. Since $g_{i}$ preserves the metric $d$ then $g_{i}^{-1} y \rightarrow x$. Using the same arguments as before we have that $h \in \widehat{\operatorname{Iso}(X)}$ hence the net $\left\{g_{i}\right\}$ converges in Iso $(X)$, a contradiction of the assumption $g_{i} \rightarrow \infty$ in $\operatorname{Iso}(X)$.

Proposition 4.5. Assume that $(\operatorname{Iso}(X), X)$ is a proper action. The following are equivalent:

(i) $\operatorname{Iso}(X)$ is Cauchy-indivisible.

(ii) Let $\left\{g_{i}\right\} \subset \operatorname{Iso}(X)$ a net with $g_{i} \rightarrow \infty$ and $\left\{g_{i} x\right\}$ be a Cauchy net for some $x \in X$. If $y \in X$ then the net $\left\{g_{i} y\right\}$ cannot have more than one limit point in the completion $\widehat{X}$ of $X$.

Proof. The direction from (i) to (ii) is trivial. If the converse implication does not hold, then there is a Cauchy net $\left\{g_{i} x\right\}$ such that there is $y \in X$, an $\varepsilon>0$, and subnets $\left\{g_{i_{k}} y\right\}$ and $\left\{g_{i_{l}} y\right\}$ of $\left\{g_{i} y\right\}$ such that $d\left(g_{i_{k}} y, g_{i_{l}} y\right) \geq \varepsilon$ for every index $k, l$. Since $\left\{g_{i} x\right\}$ is a Cauchy net in $X$ then we may assume that $d\left(g_{i_{k}} x, g_{i_{l}} x\right) \rightarrow 0$. Hence, $d\left(g_{i_{k}}^{-1} g_{i_{l}} x, x\right) \rightarrow 0$. We can define a new net $\left\{h_{i, j}\right\} \subset \operatorname{Iso}(X)$ by letting $h_{i, j}:=g_{j}^{-1} g_{i}$ for every pair of indices $(i, j)$, with direction defined by $\left(i_{1}, j_{1}\right) \leq\left(i_{2}, j_{2}\right)$ if and only if $i_{1} \leq i_{2}$ and $j_{1} \leq j_{2}$. Therefore, $h_{i_{k}, i_{l}} x \rightarrow x$. Since (Iso $\left.(X), X\right)$ is proper there is a subnet $\left\{h_{i_{k_{m}}, i_{l m}}\right\}$ and some $g \in \operatorname{Iso}(X)$ such that $h_{i_{k_{m}}, i_{l_{m}}} \rightarrow g$. Hence 
$\left\{h_{i_{k_{m}}, i_{l_{m}}} y\right\}$ is a Cauchy net in $X$, therefore for every $\varepsilon^{\prime}>0$ there exists an index $m_{0}$ such that

$$
d\left(g_{i_{k_{m}}}^{-1} g_{i_{l}} y, g_{i_{k_{n}}}^{-1} g_{i_{l}} y\right)<\varepsilon^{\prime} \quad \text { for every } m, n \geq m_{0} .
$$

By taking $m=n \geq m_{0}$ it is easy to see that $\left\{g_{i_{m}} y\right\}$ is a Cauchy net and if we follow the same procedure we can also show that $\left\{g_{i_{k_{m}}} y\right\}$ is a Cauchy net. Since $d\left(g_{i_{k_{m}}} y, g_{i_{l_{m}}} y\right) \geq \varepsilon$ for every index $m$ the net $\left\{g_{i} y\right\}$ has two limit points in the completion $\widehat{X}$ of $X$, a contradiction of our hypothesis.

\section{Cauchy-indivisible isometric actions on separable metric spaces}

In this section $(X, d)$ will denote a separable metric space such that the action (Iso $(X), X)$ is Cauchy-indivisible.

We show the adequacy of sequences in the definition of Cauchy indivisibility:

Proposition 5.1. In the definition of Cauchy indivisibility for isometric actions nets can be replaced by sequences.

Proof. Assume that if $\left\{g_{n}\right\}$ is a sequence in Iso $(X)$ such that $g_{n} \rightarrow \infty$ and $\left\{g_{n} x\right\}$ is a Cauchy sequence in $X$ for some $x \in X$ then $\left\{g_{n} x\right\}$ is a Cauchy sequence for every $x \in X$. Let $\left\{f_{i}\right\}$ be a net in $\operatorname{Iso}(X)$ such that $f_{i} \rightarrow \infty$ and $\left\{f_{i} x\right\}$ is a Cauchy net in $X$ for some $x \in X$. We will show that $\left\{f_{i} x\right\}$ is a Cauchy net in $X$ for every $x \in X$. We argue by contradiction. Suppose that there exists $y \in X$ such that $\left\{f_{i} y\right\}$ is not a Cauchy net. Hence, there is an $\varepsilon>0$ and subnets $\left\{f_{i_{k}}\right\}$ and $\left\{f_{i_{l}}\right\}$ such that $d\left(f_{i_{k}} y, f_{i_{l}} y\right) \geq \varepsilon$ for every $k, l$. Since $\left\{f_{i} x\right\}$ is a Cauchy net in $X$ there is a point $z \in \widehat{X}$ such that $\hat{f}_{i} x \rightarrow z$. Hence, the subnets $\left\{\hat{f}_{i_{k}} x\right\}$ and $\left\{\hat{f}_{i_{l}} x\right\}$ also converge to $z$. So we may find sequences $\left\{\hat{f}_{i_{k n}} x\right\}$ and $\left\{\hat{f}_{i_{l_{n}}} x\right\}$ such that $\hat{f}_{i_{k_{n}}} x \rightarrow z$ and $\hat{f}_{i_{n}} x \rightarrow z$. Therefore, $\left\{f_{i_{k_{n}}} x\right\}$ and $\left\{f_{i_{l_{n}}} x\right\}$ are Cauchy sequences in $X$ and $d\left(f_{i_{k_{n}}} y, f_{i_{l_{n}}} y\right) \geq \varepsilon$ for every $n \in \mathbb{N}$. Let $\left\{h_{n}\right\} \subset \operatorname{Iso}(X)$ with

$$
h_{4 n-3}=f_{i_{k_{2 n-1}}}, \quad h_{4 n-2}=f_{i_{l_{2 n-1}}}, \quad h_{4 n-1}=f_{i_{l_{2 n}}}, \quad \text { and } \quad h_{4 n}=f_{i_{k_{2 n}}},
$$

$n=1,2, \ldots$ It is easy to see that $\hat{h}_{n} x \rightarrow z$, hence $\left\{h_{n} x\right\}$ is a Cauchy sequence in $X$. Moreover, $\left\{h_{n} y\right\}$ is not a Cauchy sequence in $X$ since $d\left(f_{i_{k_{n}}} y, f_{i_{l_{n}}} y\right) \geq \varepsilon$ for every $n \in \mathbb{N}$ and for the same reason $h_{n} \rightarrow \infty$ in $\operatorname{Iso}(X)$, which is a contradiction of our hypothesis.

Definition 5.2. Fix a dense sequence $D=\left\{x_{i}\right\} \subset X$ in $\widehat{X}$. Since the metric $\hat{d} /(1+\hat{d})$ is an equivalent metric to $\hat{d}$ on $X$ (and also gives the same groups of isometries on $X$ and $\widehat{X}$ and the same Cauchy sequences) we may assume that $\hat{d}$ is bounded by 1 . 
We define $\delta: \operatorname{Iso}(\widehat{X}) \times \operatorname{Iso}(\widehat{X}) \rightarrow \mathbb{R}^{+}$by

$$
\delta(f, g)=\sum_{i=1}^{\infty} \frac{1}{2^{i}} \hat{d}\left(f x_{i}, g x_{i}\right)
$$

for every $f, g \in \operatorname{Iso}(\widehat{X})$. It is easy to see that $\delta$ is a left-invariant metric on $\operatorname{Iso}(\widehat{X})$.

Proposition 5.3. The uniformity of pointwise convergence, the left uniformity, and the uniformity induced by $\delta$ on $\operatorname{Iso}(\widehat{X})$ and $\operatorname{Iso}(X)$ coincide, independently of Cauchy indivisibility.

Proof. The proof is similar to the proof of [Hjorth 2008, Lemma 2.11].

Proposition 5.4. The pointwise closures of $\operatorname{Iso}(X)$ in $C(X, X)$ and of $\operatorname{Iso}(\widehat{X})$ in $C(\widehat{X}, \widehat{X})$ endowed with the metric $\delta$ are separable metric spaces.

Proof. It follows easily using the same arguments as in the proof of [Hjorth 2008, Lemma 2.11] and [Bourbaki 1966b, Chapter X, §3, Exercise 6(b), p. 327].

The following lemma will be used often in the sequel.

Lemma 5.5. Let $\left\{g_{n}\right\}$ be a sequence in $\operatorname{Iso}(X)$ such that $\left\{g_{n} x\right\}$ is a Cauchy sequence in $X$ for some $x \in X$ and $g_{n} \rightarrow \infty$. Then

(i) $\left\{g_{n} x_{n}\right\}$ is a Cauchy sequence for every Cauchy sequence $\left\{x_{n}\right\}$ in $X$ and

(ii) if $\left\{x_{k}\right\}$ is Cauchy sequence in $X$ then $\hat{g}_{n}\left[x_{k}\right] \rightarrow\left[g_{k} x_{k}\right]$ in $\widehat{X}$.

Proof. (i) The proof follows by the triangle inequality and the fact that $\left\{g_{n} x_{n_{0}}\right\}$ is a Cauchy sequence, for suitable $n_{0} \in \mathbb{N}$.

(ii) By (i), $\left\{g_{k} x_{k}\right\}$ is a Cauchy sequence in $X$, hence $\left[g_{k} x_{k}\right] \in \widehat{X}$. The rest of the proof is similar to that of (i).

Corollary 5.6. If $\left\{g_{n}\right\}$ is a sequence in Iso $(X)$ such that $g_{n} \rightarrow \infty$ andi $\left\{g_{n} x\right\}$ is a Cauchy sequence in $X$ for some $x \in X$, then $\left\{\hat{g}_{n}\right\}$ converges pointwise on $\widehat{X}$ to some $h \in C(\widehat{X}, \widehat{X})$ which preserves the metric $\hat{d}$. In addition, if $\left\{g_{n}^{-1} y\right\}$ is a Cauchy sequence for some $y \in X$, then $\left\{\hat{g}_{n}\right\}$ converges pointwise on $\widehat{X}$ to some $h \in \operatorname{Iso}(\widehat{X})$. Proof. The proof is an immediate consequence of Lemma 5.5(ii) if we set $h: \widehat{X} \rightarrow \widehat{X}$ with $h\left[x_{k}\right]:=\left[g_{k} x_{k}\right]$ for every $\left[x_{k}\right] \in \widehat{X}$.

Corollary 5.6 enables the following equivalent expressions of the corresponding sets defined in the introduction:

Notation 5.7. We have

$H=\left\{h \in C(\widehat{X}, \widehat{X}) \mid\right.$ there exists a sequence $\left\{g_{n}\right\} \subset \operatorname{Iso}(X)$ with $g_{n} \rightarrow \infty$ in $\operatorname{Iso}(X)$, $\left\{g_{n} x\right\}$ is a Cauchy sequence for some $x \in X$ and $\hat{g}_{n} \rightarrow h$ in $\left.C(\widehat{X}, \widehat{X})\right\}$. $X_{l}$ denotes the set of the limit points of the action $(\operatorname{Iso}(X), X)$ in $\widehat{X}$; specifically, 
$X_{l}=\left\{y \in \widehat{X} \mid\right.$ there exists a sequence $\left\{g_{n}\right\} \subset \operatorname{Iso}(X)$ with $g_{n} \rightarrow \infty$ in $\operatorname{Iso}(X)$, such that $\left\{g_{n} x\right\}$ is a Cauchy sequence for some $x \in X$ and $\left.y=\left[g_{k} x\right]\right\}$.

$X_{p}$ denotes the set of the special limit points of (Iso $\left.(X), X\right)$ in $\widehat{X}$; specifically, $X_{p}=\left\{y \in \widehat{X} \mid\right.$ there exists a sequence $\left\{g_{n}\right\} \subset \operatorname{Iso}(X)$ with $g_{n} \rightarrow \infty$ in $\operatorname{Iso}(X)$, such that $\left\{g_{n} x\right\}$ and $\left\{g_{n}^{-1} x\right\}$ are Cauchy sequences for some $x \in X$ and $\left.y=\left[g_{k} x\right]\right\}$.

Proposition 5.8. If $\left\{g_{n}\right\}$ is a sequence in Iso $(X)$ such that $g_{n} \rightarrow f$ on $X$ for some $f$ in $C(X, X)$, then $\hat{g}_{n} \rightarrow \hat{f}$ on $\widehat{X}$ and $\hat{f} \in \operatorname{Iso}(\widehat{X})$.

Proof. If $\left\{g_{n}\right\}$ has a convergent subsequence $\left\{g_{n_{k}}\right\}$ to some point $g \in \operatorname{Iso}(X)$ then $f=g$ on $X$ and it is easily seen that $\hat{g}_{n} \rightarrow \hat{g}$ pointwise on $\widehat{X}$.

With the notation established in the introduction, we have

Proposition 5.9. The set $E$ is

(i) the union $\widehat{\operatorname{Iso}(X)} \cup H$,

(ii) complete with respect to the uniformity of pointwise convergence on $\widehat{X}$, and

(iii) a semigroup, the Ellis semigroup of $\widehat{\operatorname{Iso}(X)}$ in $C(\widehat{X}, \widehat{X})$, that is, the pointwise closure of $\widehat{\operatorname{Iso}(X)}$ in $C(\widehat{X}, \widehat{X})$.

Proof. For part (i), take a sequence $\left\{\hat{g}_{n}\right\}$ in $\widehat{\operatorname{Iso}(X)}$ such that $\hat{g}_{n} \rightarrow h$ for some $h \in C(\widehat{X}, \widehat{X})$. If $\left\{g_{n}\right\}$ has a convergent subsequence to some $g \in \operatorname{Iso}(X)$ then, by Proposition 5.8, $h=\hat{g} \in \widehat{\operatorname{Iso}(X)}$. Let $g_{n} \rightarrow \infty$ in Iso $(X)$ and take some $x \in X$. Since $\hat{g}_{n} x \rightarrow h x$, then $\left\{g_{n} x\right\}$ is a Cauchy sequence in $X$ and therefore $h \in H$. Parts (ii) and (iii) follow from [Hjorth 2008, Lemmata 2.10 and 2.11] by noticing that a sequence $\left\{g_{n}\right\}$ in Iso $(X)$ is Cauchy with respect to the left uniformity of $\operatorname{Iso}(X)$ if and only if $\left\{g_{n} x\right\}$ is Cauchy in $X$ for every $x \in X$.

Remark 5.10. As the example in Section 6 shows, the Ellis semigroup $E$ is not, in general, a group. However:

Proposition 5.11. The Ellis semigroup $E$ is a group if and only if $X_{l}=X_{p}$.

Proof. Assume that $E$ is a group and let $y \in X_{l}$. Hence, there is a sequence $\left\{g_{n}\right\} \subset \operatorname{Iso}(X)$ with $g_{n} \rightarrow \infty$ in $\operatorname{Iso}(X)$ and a map $h \in C(\widehat{X}, \widehat{X})$ such that $\hat{g}_{n} \rightarrow h$ pointwise on $\widehat{X}$ and $y=h x$ for some $x \in X$. Since $E$ is a group then $h$ has an inverse $h^{-1}$. Thus $\hat{g}_{n}^{-1} \rightarrow h^{-1}$. The last implies that $\left\{g_{n}^{-1} x\right\}$ is a Cauchy sequence in $X$, therefore $y \in X_{p}$.

To show the converse implication, assume that $X_{l}=X_{p}$ and take some $h \in E$. By Proposition 5.9(i),

$$
h \in \widehat{\operatorname{Iso}(X)} \cup H .
$$


So, if $h \in \widehat{\operatorname{Iso}(X)}$ obviously it has an inverse. Assume that $h \in H$. Hence, there is a sequence $\left\{g_{n}\right\} \subset \operatorname{Iso}(X)$ with $g_{n} \rightarrow \infty$ in $\operatorname{Iso}(X)$ such that $\hat{g}_{n} \rightarrow h$ pointwise on $\widehat{X}$. So $\left[g_{n} x\right] \in X_{l}$ for every $x \in X$. But $X_{l}=X_{p}$, hence $\left\{g_{n}^{-1} x\right\}$ is a Cauchy sequence. Applying Corollary 5.6, $h \in \operatorname{Iso}(\widehat{X})$ so it has an inverse in $E$.

Lemma 5.12. The set $X \cup X_{l}$ is E-invariant.

Proof. It is easy to verify that $X$ and $X_{l}$ are $\widehat{\operatorname{Iso}(X)}$-invariant. We will show that they are also $H$-invariant. Let $h \in H$ and $x \in X$. By the definition of $H$ there is some sequence $\left\{g_{n}\right\}$ in Iso(X) such that $g_{n} \rightarrow \infty$ in $\operatorname{Iso}(X)$ and $\hat{g}_{n} \rightarrow h$ pointwise on $\widehat{X}$. If $\left[f_{n} x\right] \in X_{l}$, for some sequence $\left\{f_{n}\right\} \subset \operatorname{Iso}(X)$ and $x \in X$ then, by Corollary 5.6, $h\left[f_{n} x\right]=\left[g_{n} f_{n} x\right]$. If the sequence $\left\{g_{n} f_{n}\right\}$ has a convergent subsequence in Iso $(X)$ then the Cauchy sequence $\left\{g_{n} f_{n} x\right\}$ has a convergent subsequence in $X$, so it converges in $X$. So $h\left[f_{n} x\right]=\left[g_{n} f_{n} x\right] \in X$. Otherwise $g_{n} f_{n} \rightarrow \infty$ and $h\left[f_{n} x\right]=\left[g_{n} f_{n} x\right] \in X_{l}$.

Theorem 5.13. The set $X \cup X_{p}$ is the maximal subset of $X \cup X_{l}$ that contains $X$ such that the map

$$
\omega: E \times\left(X \cup X_{p}\right) \rightarrow\left(X \cup X_{p}\right) \times \widehat{X},
$$

with $\omega(f, y)=(y, f y), f \in E$, and $y \in X \cup X_{p}$, is proper.

Proof. We first show that the map $\omega: E \times\left(X \cup X_{p}\right) \rightarrow\left(X \cup X_{p}\right) \times \widehat{X}$ is proper. Since the evaluation map $E \times\left(X \cup X_{p}\right) \rightarrow \widehat{X}$ is isometric and action-like, according to Section 2, it suffices to show that the limit sets $L(x)$ are empty for every $x \in$ $X \cup X_{p}$. Let $\left\{f_{n}\right\}$ be a sequence in $E$ such that $f_{n} y \rightarrow z$ for some $y \in X \cup X_{p}$ and $z:=\left[z_{k}\right] \in \widehat{X}$.

Case I. Assume that $y \in X$. If $\left\{f_{n}\right\}$ has a subsequence $\left\{f_{n_{k}}\right\}$ in $\widehat{\operatorname{Iso}(X)}$ then either the restriction of $\left\{f_{n_{k}}\right\}$ on $X$ has a convergent subsequence in $\operatorname{Iso}(X)$ hence, by Proposition 5.8, the sequence $\left\{f_{n_{k}}\right\}$ converges pointwise to some point of $\widehat{\operatorname{Iso}(X)} \subset$ $E$, or $f_{n} \rightarrow \infty$ in Iso $(X)$. In this case, since $\left\{f_{n} y\right\}$ is a Cauchy sequence in $X$, the sequence $\left\{f_{n}\right\}$ converges pointwise to some point of $H \subset E$ by Corollary 5.6.

Assume, now, that $\left\{f_{n}\right\}$ is in $H$ and consider the dense sequence $D=\left\{x_{i}\right\}$ in $X$ which we used to define the metric $\delta$; see Definition 5.2. So, there is a sequence $\left\{x_{i_{n}}\right\}$ in $D$ such that $x_{i_{n}} \rightarrow y$. By the definition of $H$ and Proposition 5.3, there is a sequence $\left\{g_{n}\right\}$ in $\operatorname{Iso}(X)$ such that

$$
\delta\left(\hat{g}_{n}, f_{n}\right)<\frac{1}{i_{n} 2^{i_{n}}} .
$$

Hence, using the form of the metric $\delta$, we conclude that

$$
\hat{d}\left(\hat{g}_{n} x_{i_{n}}, f_{n} x_{i_{n}}\right)<\frac{1}{i_{n}} .
$$


Moreover,

$$
\begin{aligned}
\hat{d}\left(\hat{g}_{n} y, z\right) & \leq \hat{d}\left(\hat{g}_{n} x_{i_{n}}, f_{n} x_{i_{n}}\right)+\hat{d}\left(f_{n} x_{i_{n}}, f_{n} y\right)+\hat{d}\left(f_{n} y, z\right) \\
& =\hat{d}\left(\hat{g}_{n} x_{i_{n}}, f_{n} x_{i_{n}}\right)+\hat{d}\left(x_{i_{n}}, y\right)+\hat{d}\left(f_{n} y, z\right) .
\end{aligned}
$$

Therefore, $g_{n} y \rightarrow z$. Arguing as in the beginning of the proof, $\left\{g_{n}\right\}$ has a convergent subsequence to a point of $E$, hence by (5-1), the same holds for the sequence $\left\{f_{n}\right\}$.

Case II. Assume that $y \in X_{p}$. Hence, there exists a sequence $\left\{p_{k}\right\} \subset \operatorname{Iso}(X)$ with $p_{k} \rightarrow \infty$ in $\operatorname{Iso}(X)$, an isometry $h_{1} \in \operatorname{Iso}(\widehat{X})$ such that $\hat{p}_{k} \rightarrow h_{1}$ pointwise on $\widehat{X}$, and $h_{1} x:=\left[p_{k} x\right]=y$ for some $x \in X$. If $\left\{f_{n}\right\}$ has a subsequence $\left\{f_{n_{k}}\right\}$ in $\widehat{\operatorname{Iso}(X)}$ then either the restriction of $\left\{f_{n_{k}}\right\}$ on $X$ has a convergent subsequence in $\operatorname{Iso}(X)$ hence, by Proposition 5.8, the sequence $\left\{f_{n_{k}}\right\}$ converges pointwise to some point of $\widehat{\operatorname{Iso}(X)} \subset E$, or $f_{n} \rightarrow \infty$ in Iso $(X)$. If the latter holds, then we will show that there is a Cauchy sequence of the form $\left\{f_{n_{i}} p_{k_{i}} x\right\}$ in $X$ for some subsequences $\left\{f_{n_{i}}\right\}$ and $\left\{p_{k_{i}}\right\}$ of $\left\{f_{n}\right\}$ and $\left\{p_{k}\right\}$, respectively (the problem is that we do not know if $\left\{f_{n} x\right\}$ or $\left\{f_{n} p_{n} x\right\}$ is a Cauchy sequence in $X$ for some $x \in X$ ).

Let $i$ be a positive integer. Since $f_{n}\left[p_{k} x\right] \rightarrow z$ and $z:=\left[z_{k}\right] \in \widehat{X}$, there is a positive integer $n_{0}$ that depends only on $i$ such that

$$
\hat{d}\left(f_{n}\left[p_{k} x\right],\left[z_{k}\right]\right)<\frac{1}{i}
$$

for every $n \geq n_{0}(i)$. Therefore

$$
\lim _{k} d\left(f_{n} p_{k} x, z_{k}\right):=\hat{d}\left(f_{n}\left[p_{k} x\right],\left[z_{k}\right]\right)<\frac{1}{i}
$$

for every $n \geq n_{0}(i)$. Hence, using induction, we may find strictly increasing sequences of positive integers $\left\{n_{i}\right\}$ and $\left\{k_{i}\right\}$ such that

$$
d\left(f_{n_{i}} p_{k_{i}} x, z_{k_{i}}\right)<\frac{1}{i}
$$

for every positive integer $i$. Since $\left\{z_{k_{i}}\right\}$ is a Cauchy sequence then by (5-2), $\left\{f_{n_{i}} p_{k_{i}} x\right\}$ is a Cauchy sequence in $X$.

Now, either $\left\{f_{n_{i}} p_{k_{i}}\right\}$ has a convergent subsequence in $\operatorname{Iso}(X)$ (without loss of generality and for the economy of the proof we may assume that $\left\{f_{n_{i}} p_{k_{i}}\right\}$ converges in Iso $(X))$ or $f_{n_{i}} p_{k_{i}} \rightarrow \infty$ in Iso $(X)$. In both cases, by Corollary 5.6 and Proposition 5.8, there is $h_{2} \in C(\widehat{X}, \widehat{X})$ such that $\hat{f}_{n_{i}} \hat{p}_{k_{i}}=\widehat{f_{n_{i}} p_{k_{i}}} \rightarrow h_{2}$ pointwise on $\widehat{X}$. We will show that $\hat{f}_{n_{i}} \rightarrow h_{2} h_{1}^{-1}$ pointwise on $\widehat{X}$. Take $w \in \widehat{X}$. Since $h_{1} \in \operatorname{Iso}(\widehat{X})$, there is some $u \in \widehat{X}$ such that $h_{1}(u)=w$. Hence

$$
\begin{aligned}
\hat{d}\left(f_{n_{i}} w, h_{2} h_{1}^{-1} w\right) & =\hat{d}\left(f_{n_{i}} h_{1} u, h_{2} u\right) \leq \hat{d}\left(f_{n_{i}} h_{1} u, \widehat{f_{n_{i}} p_{k_{i}}} u\right)+\hat{d}\left(\widehat{f_{n_{i}} p_{k_{i}}} u, h_{2} u\right) \\
& =\hat{d}\left(h_{1} u, \hat{p}_{k_{i}} u\right)+\hat{d}\left(\widehat{f_{n_{i}} p_{k_{i}}} u, h_{2} u\right),
\end{aligned}
$$


which converges to 0 , since $\hat{p}_{k_{i}} \rightarrow h_{1}$ and $\widehat{f_{n_{i}} p_{k_{i}}} \rightarrow h_{2}$ pointwise on $\widehat{X}$. Hence $\left\{f_{n} x\right\}$ is a Cauchy sequence for every $x \in X$. Since we assumed that $f_{n} \rightarrow \infty$ in Iso $(X)$ then, by Corollary 5.6, $\left\{f_{n}\right\}$ converges pointwise on $\widehat{X}$ to $h_{2} h_{1}^{-1} \in E$.

To finish the proof of the second case assume that $\left\{f_{n}\right\}$ is in $H$. Then arguing as in the first case we can show that $\left\{f_{n}\right\}$ has a convergent subsequence to a point of $E$.

Next, we show that if $Y$ is a subset of $X \cup X_{l}$ that contains $X$ such that the map

$$
\omega: E \times Y \rightarrow Y \times \widehat{X}
$$

is proper then $Y \subset X \cup X_{p}$. To see that take a point $\left[g_{k} x\right] \in Y \backslash X$. This means that $\left\{g_{k}\right\}$ is a sequence in $\operatorname{Iso}(X)$ such that $g_{k} \rightarrow \infty$ in $\operatorname{Iso}(X)$ and $\left\{g_{k} x\right\}$ is a Cauchy sequence in $X$. By Lemma 5.5(ii), $\hat{g}_{n} x \rightarrow\left[g_{k} x\right]$ and, by Corollary 5.6, $\left\{\hat{g}_{n}\right\}$ converges pointwise on $\widehat{X}$ to some $h \in C(\widehat{X}, \widehat{X})$. Note that $x \in X \subset Y$. Since $\hat{g}_{n} x \rightarrow\left[g_{k} x\right]$ and $\hat{g}_{n}$ preserves the metric $\hat{d}$ then $\hat{g}_{n}^{-1}\left[g_{k} x\right] \rightarrow x$. Hence, by the properness of $\omega$, we may assume that $\left\{\hat{g}_{n}^{-1}\right\}$ has a subsequence $\left\{\hat{g}_{n_{k}}^{-1}\right\}$ that converges pointwise to some $f \in E$. This makes $h$ a surjection, hence $h \in \operatorname{Iso}(\widehat{X})$. Therefore, $\left[g_{k} x\right] \in X_{p}$, so $Y \backslash X \subset X_{p}$.

Note that, as the following example shows, it may happen that $X_{p}=X_{l} \neq \varnothing$, $X \cup X_{p} \neq \widehat{X}$, and the set $X \cup X_{p}$ is not the maximal subset of $\widehat{X}$ such that the action $\left(E, X \cup X_{p}\right)$ is proper.

Example 5.14. Take

$$
X:=\{(x, y) \in \mathbb{R} \mid x \in \mathbb{Q}+\sqrt{2} \mathbb{N}, y>0\},
$$

endowed with the Euclidean metric. Its group of isometries is the additive group of the rational numbers acting by horizontal translations. Therefore, (Iso $(X), X)$ is Cauchy-indivisible. Obviously $\widehat{X}$ is the closed upper half-plane, $X_{p}=X_{l} \neq \varnothing$, $X \cup X_{p}$ is the open upper half-plane, and $E$ is the additive group of the real numbers acting by horizontal translations on $\widehat{X}$. Hence $E$ acts properly on $\widehat{X}$.

Remark 5.15. The sets $X_{p}$ and $X_{l}$ constructed in Theorem 5.13 are optimal in the sense that if one may think to replace the sets $X_{p}$ and $X_{l}$ with the following more general sets

$X_{l}^{*}=\left\{y \in \widehat{X} \mid\right.$ there exists a sequence $\left\{g_{n}\right\} \subset \operatorname{Iso}(X)$ and some $x \in X$ such that $g_{n} \rightarrow \infty$ in Iso $(X),\left\{g_{n} x\right\}$ is a Cauchy sequence, and $y=\left[g_{k} x_{k}\right]$, for some $\left.\left[x_{k}\right] \in \widehat{X}\right\}$ 
and

$X_{p}^{*}=\left\{y \in \widehat{X} \mid\right.$ there exists a sequence $\left\{g_{n}\right\} \subset \operatorname{Iso}(X)$ and some $x \in X$ such that $g_{n} \rightarrow \infty$ in $\operatorname{Iso}(X),\left\{g_{n} x\right\}$ and $\left\{g_{n}^{-1} x\right\}$ are Cauchy sequences,

$$
\text { and } \left.y=\left[g_{k} x_{k}\right] \text {, for some }\left[x_{k}\right] \in \widehat{X}\right\} \text {, }
$$

and ask if the set $X \cup X_{p}^{*}$ is the maximal subset of the completion $\widehat{X}$ such that the map $\omega^{*}: E \times\left(X \cup X_{p}^{*}\right) \rightarrow\left(X \cup X_{p}^{*}\right) \times \widehat{X}$ with $\omega^{*}(f, y)=(y, f y), f \in E$, and $y \in X \cup X_{p}^{*}$ is proper this is not true in general. This follows from the following assertion and Example 5.17, which shows that there is a metric space $X$ such that (Iso $(X), X)$ is Cauchy-indivisible, $X_{p} \neq \varnothing$, and the map $\omega^{*}$ as above is not proper.

Assertion 5.16. If $X_{p}^{*} \neq \varnothing$ (equivalently $X_{p} \neq \varnothing$ ) then $X_{p}^{*}=\widehat{X}$.

Proof. Let $y=\left[x_{k}\right]$ in $\widehat{X}$. By assumption, there exists a sequence $\left\{g_{n}\right\} \subset \operatorname{Iso}(X)$ and a point $x \in X$ such that $g_{n} \rightarrow \infty$ in $\operatorname{Iso}(X)$ and the sequences $\left\{g_{n} x\right\}$ and $\left\{g_{n}^{-1} x\right\}$ are Cauchy. By Lemma 5.5, $\left\{g_{n} x_{n}\right\}$ is a Cauchy sequence in $X$ and $g_{k}^{-1}\left[g_{k} x_{k}\right] \rightarrow$ $\left[g_{k}^{-1} g_{k} x_{k}\right]=\left[x_{k}\right]=y$. Hence, $y \in X_{p}^{*}$.

Example 5.17. This is a combination of Example 4.3 and of a 3 -dimensional variation of the "river metric" [Engelking 1989, Example 4.1.6]. Let

$$
X=\{(x, y, z) \mid x \in \mathbb{Q}+\sqrt{2} \mathbb{N}, y \in \mathbb{Q}+\sqrt{2} \mathbb{N}, z>0\} .
$$

For every pair of points $w_{1}=\left(x_{1}, y_{1}, z_{1}\right), w_{2}=\left(x_{2}, y_{2}, z_{2}\right) \in X$ define

$$
d\left(w_{1}, w_{2}\right):= \begin{cases}\left|y_{1}-y_{2}\right|+\left|z_{1}-z_{2}\right|, & \text { if } x_{1}=x_{2}, \\ \left|y_{1}\right|+\left|y_{2}\right|+\left|x_{1}-x_{2}\right|+\left|z_{1}-z_{2}\right|, & \text { if } x_{1} \neq x_{2} .\end{cases}
$$

We can easily verify that $d$ is a metric on $X$. The group of isometries $\operatorname{Iso}(X, d)$ consists of all the maps $g: X \rightarrow X$ of the form

$$
g(x, y, z)=(x+p, y+q, z), \quad p, q \in \mathbb{Q} .
$$

The action (Iso $(X), X)$ is Cauchy-indivisible since $X$ does not contain the $x y$-plane (the last coordinate of the points of $X$ is positive). Then

$$
X_{p}=\{(x, y, z) \mid x \in \mathbb{Q}+\sqrt{2} \mathbb{N}, y \in \mathbb{R}, z>0\} .
$$

To see that take $x \in \mathbb{Q}+\sqrt{2} \mathbb{N}, y \in \mathbb{R}$, and $z>0$ and choose $k \in \mathbb{N}$ such that $y-\sqrt{2} k \notin$ $\mathbb{Q}$. Let $\left\{q_{n}\right\}$ be a sequence of rational numbers such that $q_{n} \rightarrow y-\sqrt{2} k$. Hence, if we let $\left\{g_{n}\right\} \subset \operatorname{Iso}(X)$ with $g_{n}(x, y, z):=\left(x, y+q_{n}, z\right)$ then $g_{n}(x, \sqrt{2} k, z)=$ $\left(x, q_{n}+\sqrt{2} k, z\right) \rightarrow(x, y, z)$. Hence $(x, y, z) \in X_{p}$. Observe that

$$
\widehat{X}=\{(x, y, z) \mid x \in \mathbb{Q}+\sqrt{2} \mathbb{N}, y \in \mathbb{R}, z \geq 0\},
$$


and $E$ consists of all the maps $g: \widehat{X} \rightarrow \widehat{X}$ with

$$
g(x, y, z)=(x+p, y+r, z), \quad p \in \mathbb{Q}, \quad r \in \mathbb{R} .
$$

However, the map $\widehat{\omega}: E \times \widehat{X} \rightarrow \widehat{X} \times \widehat{X}$ with $\widehat{\omega}(f, w)=(w, f w), f \in E$, and $w \in \widehat{X}$ is not proper since if we take a sequence of rational numbers $\left\{p_{n}\right\}$ such that $p_{n} \rightarrow \sqrt{2}$ and let $\left\{g_{n}\right\} \subset E$ with $g_{n}(x, y, z)=\left(x+p_{n}, y, z\right)$ then $g_{n}(x, 0,0) \rightarrow(x+\sqrt{2}, 0,0)$ for each $x \in \mathbb{Q}+\sqrt{2} \mathbb{N}$. The sequence $\left\{g_{n}\right\}$ diverges in $E$ since, for instance, the distance of the points $g_{n}(\sqrt{2}, \sqrt{2}, 1)=\left(q_{n}+\sqrt{2}, \sqrt{2}, 1\right)$ from any point of $X$ is eventually at least $\sqrt{2}$. Hence the limit set $L((x, 0,0))$ is not empty.

A question that arises naturally from Theorem 5.13 is if the action of the Ellis semigroup $E$ on $X \cup X_{l}$ is proper. Surprisingly, as the following proposition shows, this is equivalent to the existence of a Weil completion (with respect to the uniformity of pointwise convergence) for the group Iso $(X)$. But first let us recall a few things about the Weil completion of Iso $(X)$, defined in the introduction. The uniformity of pointwise convergence on $X$ coincides with the left uniformity of Iso $(X)$ (see [Bourbaki 1966a, Chapter III, §3.1 and Chapter X, §3, Exercise 19(a), p. 332]) and Iso $(X)$ has Weil completion with respect to this uniformity if the left and the right uniformities coincide; see [Bourbaki 1966a, Chapter III, §3.4 and §3, Exercise 3, p. 306]. Note that the left completion of $\operatorname{Iso}(X)$ does not depend on the choice of a left-invariant metric on Iso $(X)$; see [Hjorth 2008, Lemma 2.9].

Proposition 5.18. The following are equivalent:

(i) The map $\omega: E \times\left(X \cup X_{l}\right) \rightarrow\left(X \cup X_{l}\right) \times \widehat{X}$ is proper.

(ii) $E$ is a group (precisely a closed subgroup of $\operatorname{Iso}(\widehat{X})$ ).

(iii) Iso( $X)$ has a Weil completion with respect to the uniformity of pointwise convergence (in this case $E$ is the Weil completion of $\operatorname{Iso}(X)$ ).

Proof. We show that (i) implies (ii) and vice versa. Suppose that $\omega$ is proper. Take some $h \in E$. Since $E$ is a semigroup, see Proposition 5.9(iii), we have only to show that $h$ has an inverse in $E$. If $h=\hat{g} \in \widehat{\operatorname{Iso}(X)}$ for some $g \in \operatorname{Iso}(X)$, then $\hat{g}^{-1}$ is the inverse of $h$ in $\widehat{\operatorname{Iso}(X)} \subset E$. If $h \in H$ there is a sequence $\left\{g_{n}\right\}$ in Iso( $\left.X\right)$ such that $g_{n} \rightarrow \infty$ in Iso $(X)$ and $\hat{g}_{n} \rightarrow h$ pointwise on $\widehat{X}$. Hence, if $x \in X$ then $\hat{g}_{n} x \rightarrow h x$. Since $\hat{g}_{n}$ preserves the metric $\hat{d}$ then $\hat{g}_{n}^{-1} h x=\hat{g}_{n}^{-1} h x \rightarrow x$. By Lemma 5.12, $h x \in X \cup X_{l}$, hence, by the properness of $\omega,\left\{\hat{g}_{n}^{-1}\right\}$ has a convergent subsequence $\left\{\hat{g}_{n_{k}}^{-1}\right\}$ to some $f \in E$. This makes $h$ a surjection, hence $h \in \operatorname{Iso}(\widehat{X})$ and $h$ has an inverse in $E$. To show the converse implication note that if $E$ is a group then $X_{l}=X_{p}$; see Proposition 5.11. Hence, by Theorem 5.13, the map $\omega$ is proper.

To finish the proof of the proposition let us show that (iii) implies (ii) and vice versa. Note that Iso $(X)$ has a Weil completion if and only if the map with $g \mapsto g^{-1}$ for every $g \in \operatorname{Iso}(X)$ maps Cauchy sequences of $\operatorname{Iso}(X)$ to Cauchy sequences; see 
[Bourbaki 1966a, Chapter III, §3.4, Theorem 1]. It is easy to check that in the case when $\operatorname{Iso}(X)$ is Cauchy-indivisible this is equivalent to $X_{l}=X_{p}$. Equivalently, by Proposition 5.11, $E$ is a group.

Remark 5.19. In the case when $\operatorname{Iso}(X)$ is a locally compact group, for example, if $X$ is a locally compact space and $\operatorname{Iso}(X)$ acts properly on it (as it is known in the case $X$ is connected), then by [Bourbaki 1966a, Chapter III, §3, Exercise 8, p. 307], Iso $(X)$ has a locally compact completion hence $E$ is a locally compact group.

We summarize with the following.

Corollary 5.20. If $E$ is a group the action (Iso $(X), X)$ is embedded densely in the proper action $\left(E, X \cup X_{l}\right)$ such that the following equivariant diagram commutes:

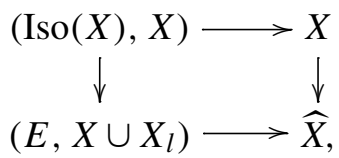

where $X \rightarrow X \cup X_{l}$ is the inclusion map and the map $\operatorname{Iso}(X) \rightarrow E$ is defined by $g \mapsto \hat{g}$ for every $g \in \operatorname{Iso}(X)$. By “densely" we mean that $X$ is dense in $X \cup X_{l}$ and $\widehat{\operatorname{Iso}(X)}$ is dense in $E$.

Question 5.21. The above embedding of a Cauchy-indivisible action as a dense subaction of a proper one establishes a remarkable connection between Cauchyindivisible and proper actions, and at the same time proposes an interesting question: Is there any analogy with the situation of embedding of a proper action (on a locally compact and connected space) in an appropriate zero-dimensional compactification, like in [Abels 1972; Manoussos and Strantzalos 2007]? Namely, can we obtain any structurally informative correspondence between divergent nets in Iso $(X)$ and suitable subsets of $X_{l}$ ?

Remark 5.22. As we will see in the example described in Section 6 it may happen that $X_{p} \neq X_{l}$ and $X \cup X_{l}=\widehat{X}$.

In view of possible questions for refinements of Corollary 5.20 we note that it may happen that $X \cup X_{p}=\widehat{X}$ and $E$ is not dense in Iso $(\widehat{X})$, as the following example shows:

Example 5.23. There is a separable metric space $(X, d)$ such that $(\operatorname{Iso}(X), X)$ is Cauchy-indivisible, proper, $X \cup X_{p}=\widehat{X}$, and $\operatorname{Iso}(X)$ has a Weil completion which does not coincide with the group $\operatorname{Iso}(\widehat{X})$.

Proof. We let $X$ be the set $\mathbb{Q}+\sqrt{2} \mathbb{N}$ endowed with the Euclidean metric; see Example 4.3. It is easy to check that $X \cup X_{p}=X \cup X_{l}=\mathbb{R}$, see also Example 5.17, hence by Propositions 5.11 and 5.18, Iso $(X)$ has a Weil completion (or just observe that Iso $(X)$ is an abelian group and use [Bourbaki 1966a, Chapter III, §3.5, 
Theorem 2]). But all the reflections of the space are excluded, hence the pointwise closure $E$ of $\widehat{\operatorname{Iso}(X)}$ does not coincide with Iso $(\mathbb{R})$.

\section{An example of a proper Cauchy-indivisible action of a group which has no Weil completion}

In this section we show that there is a separable metric space $X$ such that the action (Iso $(X), X)$ is proper and Cauchy-indivisible, and the Ellis semigroup $E$ is not a group. Equivalently, in view of Proposition 5.18, Iso $(X)$ has no Weil completion. Consider the space of the integers $\mathbb{Z}$ with the discrete metric $d$, that is, if $m, n \in \mathbb{Z}$ then $d(m, n)=0$ if $m=n$ and $d(m, n)=1$ otherwise. The group of isometries Iso $(\mathbb{Z})$ consists of all the self bijections of $\mathbb{Z}$ and is an example of a topological group that has no Weil completion. To see that take $f_{n}: \mathbb{Z} \rightarrow \mathbb{Z}$ with $f_{n} z=z$ for $-n<z<0, f_{n}(-n)=0$, and $f_{n} z=z+1$ otherwise. Then it is easy to verify that $f_{n} \rightarrow f$, where $f z=z$ for $z<0$, and $f z=z+1$ for $z \geq 0$. Hence $\left\{f_{n} z\right\}$ is a Cauchy sequence in $\mathbb{Z}$ for every $z \in \mathbb{Z}$, therefore $\left\{f_{n}\right\}$ is a Cauchy sequence in Iso( $\left.\mathbb{Z}\right)$ with respect to the uniformity of pointwise convergence on $\mathbb{Z}$. But $\left\{f_{n}^{-1} 0\right\}=\{-n\}$ is not a Cauchy sequence, so neither is $\left\{f_{n}^{-1}\right\}$. Thus, by [Bourbaki 1966a, Chapter

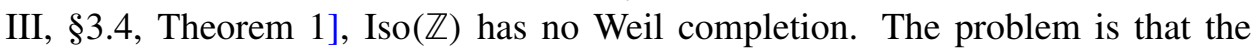
action $(\operatorname{Iso}(\mathbb{Z}), \mathbb{Z})$ is not Cauchy-indivisible. To see that notice that $\left\{f_{n}^{-1} 1\right\}=\{0\}$ but $\left\{f_{n}^{-1} 0\right\}=\{-n\}$ is not a Cauchy sequence. Nevertheless, the group $\operatorname{Iso}(\operatorname{Iso}(\mathbb{Z}))$ is Cauchy-indivisible and has no Weil completion as we show in the following.

Take an enumeration $A=\left\{z_{i}\right\}$ of $\mathbb{Z}$ and equip Iso( $\left.\mathbb{Z}\right)$ with the metric

$$
\varrho(f, g)=\sum_{i=1}^{\infty} \frac{1}{3^{i}} d\left(f z_{i}, g z_{i}\right)
$$

for $f, g \in \operatorname{Iso}(\mathbb{Z})$. In view of Proposition 5.3 the uniformity of pointwise convergence, the left uniformity, and the uniformity induced by $\varrho$ on Iso(ZZ) coincide (the choice of $\frac{1}{3}$ instead of $\frac{1}{2}$ in Definition 5.2 will be clarified in the proof of Lemma 6.1). Note that (Iso( $\mathbb{Z}), \varrho$ ) is a separable metric space. We will show that $\operatorname{Iso}(\operatorname{Iso}(\mathbb{Z}))$ is Cauchy-indivisible but has no Weil completion.

Lemma 6.1. If $T \in \operatorname{Iso}(\operatorname{Iso}(\mathbb{Z}))$ and $f, g \in \operatorname{Iso}(\mathbb{Z})$ then

$$
d(T(f) z, T(g) z)=d(f z, g z)
$$

for every $z \in \mathbb{Z}$.

Proof. Since $\varrho(T(f), T(g))=\varrho(f, g)$ then

$$
\sum_{i=1}^{\infty} \frac{1}{3^{i}} d\left(T(f) z_{i}, T(g) z_{i}\right)=\sum_{i=1}^{\infty} \frac{1}{3^{i}} d\left(f z_{i}, g z_{i}\right) .
$$


Since the values of $d$ are 0 or 1 then $d\left(T(f) z_{n}, T(g) z_{n}\right)=d\left(f z_{n}, g z_{n}\right)$, for every $z_{n} \in A=\mathbb{Z}$ (here is the role of the choice of $\frac{1}{3}$ instead of $\frac{1}{2}$ ).

Proposition 6.2. If $T \in \operatorname{Iso}(\operatorname{Iso}(\mathbb{Z}))$ and $f \in \operatorname{Iso}(\mathbb{Z})$ then $T(f)=T(e) \circ f$, where $e$ is the unit element of $\operatorname{Iso}(\mathbb{Z})$.

Proof. Let $z_{k}$ and $z_{l}$ be two distinct integers and let $g \in \operatorname{Iso}(\mathbb{Z})$ be such that $g z_{k}=z_{l}$, $g z_{l}=z_{k}$, and $g z=z$ elsewhere. We show that $T(g)=T(e) \circ g$. If $z \neq z_{k}, z_{l}$ then, by Lemma 6.1, $d(T(g) z, T(e) z)=d(g z, z)=0$. Hence $T(g) z=T(e) z=T(e) \circ g z$. Moreover

$$
d\left(T(g) z_{k}, T(e) z_{k}\right)=d\left(g z_{k}, z_{k}\right)=d\left(z_{l}, z_{k}\right)=1
$$

and, similarly, $d\left(T(g) z_{l}, T(e) z_{l}\right)=1$. Since $T(g) z_{k} \neq T(g) z=T(e) z$ for $z \neq$ $z_{k}, z_{l}$ and $T(e)$ is surjective then $T(g) z_{k}=T(e) z_{l}=T(e) \circ g z_{k}$ and, similarly, $T(g) z_{l}=T(e) \circ g z_{l}$. Therefore $T(g)=T(e) \circ g$.

Fix $f \in \operatorname{Iso}(\mathbb{Z})$ and some $z \in \mathbb{Z}$. If $f z=z$ then $T(f) z=T(e) z=T(e) \circ f z$ since $d(T(f) z, T(e) z)=d(f z, z)=0$. If $f z \neq z$, let $g \in \operatorname{Iso}(\mathbb{Z})$ with $g z=f z$, $g f z=z$, and $g w=w$ elsewhere. Since $d(T(f) z, T(g) z)=d(f z, g z)=0$ then $T(f) z=T(g) z$. Using the result of the previous paragraph, $T(f) z=T(g) z=$ $T(e) \circ g z=T(e) \circ f z$. Since $z$ was arbitrary then $T(f)=T(e) \circ f$.

Corollary 6.3. Let $L, T \in \operatorname{Iso}(\operatorname{Iso}(\mathbb{Z}))$. Then $L \circ T(e)=L(e) \circ T(e)$ and $T^{-1}(e)=$ $(T(e))^{-1}$.

Proof. Since $T(f)=T(e) \circ f$ for every $T \in \operatorname{Iso}(\operatorname{Iso}(\mathbb{Z}))$ and $f \in \operatorname{Iso}(\mathbb{Z})$, then

$$
L \circ T(f)=L(T(f))=L(e) \circ T(f)=L(e) \circ T(e) \circ f .
$$

Hence, $L(e) \circ T(e)=L \circ T(e)$. If $I$ denotes the identity on $\operatorname{Iso}(\operatorname{Iso}(\mathbb{Z}))$, then $f=I(f)=I(e) \circ f$. Hence $I(e)=e$ and $T^{-1}(e)=(T(e))^{-1}$.

Proposition 6.4. The map $\mathscr{B}: \operatorname{Iso}(\operatorname{Iso}(\mathbb{Z})) \rightarrow \operatorname{Iso}(\mathbb{Z})$ with $\mathscr{B}(T)=T(e)$ is a uniform group isomorphism with respect to the uniformities of pointwise convergence on the underlying spaces $\mathrm{Iso}(\mathbb{Z})$ and $\mathbb{Z}$, respectively.

Proof. By Proposition 5.3 we can equip Iso(Iso $(\mathbb{Z})$ ) with a left-invariant metric $\sigma$ such that the uniformity of pointwise convergence, the left uniformity, and the uniformity induced by $\sigma$ on $\operatorname{Iso}(\operatorname{Iso}(\mathbb{Z}))$ coincide. Let $L_{n}, T_{n} \in \operatorname{Iso}(\operatorname{Iso}(\mathbb{Z}))$ such that $\sigma\left(L_{n}, T_{n}\right) \rightarrow 0$, hence $\sigma\left(T_{n}^{-1} L_{n}, I\right) \rightarrow 0$. Therefore $T_{n}^{-1} L_{n} \rightarrow I$ pointwise on Iso( $\mathbb{Z})$ so $T_{n}^{-1} L_{n}(e) \rightarrow e$, thus $\varrho\left(L_{n}(e), T_{n}(e)\right) \rightarrow 0$. For the converse, note that if $\varrho\left(T_{n}^{-1} L_{n}(e), e\right) \rightarrow 0$ then $\varrho\left(T_{n}^{-1} L_{n}(e) \circ f, f\right) \rightarrow 0$ for every $f \in \operatorname{Iso}(\mathbb{Z})$ since the $\operatorname{map} \operatorname{Iso}(\mathbb{Z}) \rightarrow \operatorname{Iso}(\mathbb{Z})$ with $g \mapsto g f$ is continuous. Hence $T_{n}^{-1} L_{n} \rightarrow I$ pointwise on Iso( $\mathbb{Z})$. Corollary 6.3 implies that $\mathscr{B}$ is also group isomorphism.

Proposition 6.5. The group $\operatorname{Iso}(\operatorname{Iso}(\mathbb{Z}))$ is Cauchy-indivisible and has no Weil completion. 
Proof. Let us show firstly that Iso(Iso( $\mathbb{Z})$ ) is Cauchy-indivisible. Let $\left\{T_{n}\right\} \subset$ $\operatorname{Iso}(\operatorname{Iso}(\mathbb{Z}))$ and $f \in \operatorname{Iso}(\mathbb{Z})$ such that $\left\{T_{n}(f)\right\}$ is a Cauchy sequence in $\operatorname{Iso}(\mathbb{Z})$. Take some $g \in \operatorname{Iso}(\mathbb{Z})$. Since $\left\{T_{n}(f)\right\}$ is a Cauchy sequence in Iso(ZZZ) then it is easy to see that $\left\{T_{n}(f) z\right\}$ is a Cauchy sequence for every $z \in \mathbb{Z}$. Equivalently, $\left\{T_{n}(f) f^{-1} g z\right\}$ is a Cauchy sequence for every $z \in \mathbb{Z}$. By Proposition 6.2,

$$
T_{n}(f) f^{-1} g z=T_{n}(e) \circ f f^{-1} g z=T_{n}(e) \circ g z=T_{n}(g) z .
$$

Therefore $\left\{T_{n}(g)\right\}$ is a Cauchy sequence in $\operatorname{Iso}(\operatorname{Iso}(\mathbb{Z}))$ for every $g \in \operatorname{Iso}(\mathbb{Z})$, hence $\operatorname{Iso}(\operatorname{Iso}(\mathbb{Z}))$ is Cauchy-indivisible.

Since by the previous proposition the groups $\operatorname{Iso}(\operatorname{Iso}(\mathbb{Z}))$ and Iso( $\mathbb{Z})$ are uniformly isomorphic and the group Iso $(\mathbb{Z})$ has no Weil completion then the same also holds for $\operatorname{Iso}(\operatorname{Iso}(\mathbb{Z}))$.

Proposition 6.6. The action $(\operatorname{Iso}(\operatorname{Iso}(\mathbb{Z}))$, Iso( $\mathbb{Z}))$ is proper.

Proof. Let $f, g \in \operatorname{Iso}(\mathbb{Z})$ and $\left\{T_{n}\right\} \subset \operatorname{Iso}(\operatorname{Iso}(\mathbb{Z}))$ be a sequence such that $T_{n}(f) \rightarrow g$. Hence, by Proposition 6.2, $T_{n}(e) \circ f \rightarrow g$ thus $T_{n}(e) \rightarrow g f^{-1}$. Therefore $\left\{T_{n}(h)\right\}$ converges for every $h \in \operatorname{Iso}(\mathbb{Z})$. Since $\left(T_{n}(e)\right)^{-1} \rightarrow f g^{-1}$ it is easy to verify that $\left\{T_{n}\right\}$ converges in $\operatorname{Iso}(\operatorname{Iso}(\mathbb{Z}))$ hence the action (Iso(Iso $\left.(\mathbb{Z})\right)$, Iso(ZZZ)) is proper.

Remark 6.7. Notice that $\operatorname{Iso}(\operatorname{Iso}(\mathbb{Z}))$ is not locally compact since it has no Weil completion $(\operatorname{Iso}(\mathbb{Z})$ is, of course, not locally compact).

\section{Borel sections, fundamental sets, and Cauchy indivisibility}

As it is indicated in the introduction a section of an action $(G, X)$ is a subset of $X$ which contains only one point from each orbit. If a section is a Borel subset of $X$ it called a Borel section. Concerning the existence of Borel sections, if $(Y, d)$ is a separable metric space and $\mathscr{R}$ is an equivalence relation on $Y$ such that the $\mathscr{R}$-saturation of each open set is Borel, then there is a Borel set $S$ whose intersection with each $\mathscr{R}$-equivalence class which is complete with respect to $d$ is nonempty, and whose intersection with each $\mathscr{R}$-equivalence class is at most one point; see [Kallman and Mauldin 1978, Lemma 2]. The problem of the existence of a Borel section for a continuous Polish action is of remarkable significance because the existence of a Borel section is equivalent to many interesting facts, like that the underlying space has only trivial ergodic measures and that the orbit space has a standard Borel structure and has no nontrivial atoms. Recall that an action $(G, X)$ is called Polish if both $G$ and $X$ are Polish spaces, that is, they are separable and metrizable by a complete metric. Keeping the previous in mind we have the following:

Proposition 7.1. If the Ellis semigroup $E$ is a group then the action $\left(E, X \cup X_{l}\right)$ has a Borel section. 
Proof. Assume that the Ellis semigroup $E$ is a group. Since by Proposition 5.11 we have $X_{l}=X_{p}$ and by Proposition 5.18 the map $\omega: E \times\left(X \cup X_{l}\right) \rightarrow\left(X \cup X_{l}\right) \times \tilde{X}$ is proper then each orbit $E x, x \in X \cup X_{l}$, is closed in $\widetilde{X}$. Hence, by [Kallman and Mauldin 1978, Lemma 2] there exists a Borel set $S \subset \widetilde{X}$ such that $S \cap\left(X \cup X_{l}\right)$ is a Borel section (with respect to the relative topology of $X \cup X_{l}$ ) for the action $\left(E, X \cup X_{l}\right)$.

A very useful notion in the theory of proper actions on locally compact spaces with paracompact orbit spaces is the notion of a fundamental set.

Let $G$ be a topological group which acts continuously on a topological space $X$ and $A, B \subset X$. Let us call the set $G_{A B}:=\{g \in G: g A \cap B \neq \varnothing\}$ the transporter from $A$ to $B$.

Definition 7.2. A subset $F$ of $X$ is called a fundamental set for the action $(G, X)$ if the following holds.

(i) $G F=X$.

(ii) For every $x \in X$ there exists a neighborhood $V \subset X$ of $x$ such that the transporter $G_{V F}$ of $V$ to $F$ has compact closure in $G$.

For locally compact spaces we can replace condition (ii) with the following equivalent condition.

(iia) The transporter $G_{K F}$ from $K$ to $F$ has compact closure in $G$ for every nonempty compact subset $K$ of $X$.

Note that the existence of a fundamental set implies that the action group $G$ is locally compact and the action $(G, X)$ is proper.

The notion of a fundamental set is relative to the notion of a section but it is different in general, in the sense that there are cases where a section is a fundamental set, cases where a fundamental set fails to be a section, and cases where a section fails to be a fundamental set. A section may not be Borel or even if it is Borel may not be contained in any fundamental set, as the following example shows.

Example 7.3. The action $(\mathbb{Z}, \mathbb{R})$ with $(z, r) \mapsto r+z, z \in \mathbb{Z}, r \in \mathbb{R}$, is proper and it has a Borel section which is not contained in any fundamental set. Indeed, it is easy to see that the set

$$
S:=\left([0,1) \backslash \bigcup_{n \in \mathbb{N}}\left\{\frac{1}{n}\right\}\right) \cup \bigcup_{n \in \mathbb{N}}\left\{n+\frac{1}{n}\right\}
$$

is a section because the interval $[0,1)$ is a section (and a fundamental set) for the action $(\mathbb{Z}, \mathbb{R})$. Take an open ball $B$ centered at 0 with radius $\varepsilon>0$. Then there exists $n_{0} \in \mathbb{N}$ such that $1 / n<\varepsilon$ for every $n \geq n_{0}$. Let $A$ be a subset of $\mathbb{R}$ that contains $S$. Hence $\left\{n \mid n \geq n_{0}\right\}$ is a subset of the transporter $\mathbb{Z}_{B S} \subset \mathbb{Z}_{B A}$, so $A$ cannot be a fundamental set. 
It is also possible to construct a section which is not Borel. Take a set $D \subset[0,1)$ which is not a Borel set and consider the set $S_{1}:=D \cup\{x+2 \mid x \in \mathbb{R} \backslash D\}$. Obviously $S_{1}$ is a section which is not a Borel subset of the reals.

Nevertheless sections, Borel sections, and fundamental sets have a very strong connection as the following theorem shows.

Theorem 7.4. Let $G$ be a group which acts properly on a locally compact space $X$, and suppose that the orbit space $G \backslash X$ is paracompact. Let $S$ be a section for the action $(G, X)$. Then

(i) For every open neighborhood $U$ of $S$ we can construct a closed fundamental set $F_{c}$ and an open fundamental set $F_{o}$ such that $F_{c} \subset F_{o} \subset U$.

(ii) If in addition, $(X, d)$ is a separable metric space, in which case the action $(G, X)$ is Cauchy-indivisible, by Theorem 3.3, then there exists a Borel section $S_{B}$, which is also a fundamental set, such that $S_{B} \subset F_{c} \subset F_{o} \subset U$.

Proof. (i) Since $U$ is open it is a union of open balls, let us say $S_{i}, i \in I$. Let $p: X \rightarrow G \backslash X$ be the natural map $x \mapsto G x$. Then $p\left(S_{i}\right), i \in I$, is an open covering of the locally compact and paracompact space $G \backslash X$. Hence, there is a locally finite refinement $\left\{W_{j}\right\}, j \in J$, which consists of open subsets of $G \backslash X$ with compact closures such that $W_{j} \subset p\left(S_{i_{j}}\right)$, for some $i_{j} \in I$. Now we can follow the classical proof for the existence of fundamental sets; see [Koszul 1965, Lemma 2, p. 8]. Let $\left\{V_{j}\right\}$ be an open covering of $G \backslash X$ such that $\bar{V}_{j} \subset W_{j}$ for every $j \in J$. Fix an index $j \in J$ and consider the restriction of the natural map $p: X \rightarrow G \backslash X$ on the open ball $S_{i_{j}}$. Since $S_{i_{j}}$ is locally compact then there exists an open set $U_{i_{j}} \subset S_{i_{j}}$ with compact closure and a compact set $K_{i_{j}} \subset U_{i_{j}} \subset S_{i_{j}}$ such that $p\left(U_{i_{j}}\right)=W_{j}$ and $p\left(K_{i_{j}}\right)=\bar{V}_{j}$. Let $F_{c}:=\bigcup_{j} K_{i_{j}}$ and $F_{o}:=\bigcup_{j} U_{i_{j}}$. The family $\left\{U_{i_{j}}\right\}_{j \in J}$ is locally finite in $X$ hence the set $F_{c}$ is closed; see [Bourbaki 1966a, Chapter I, $\S 1.5$, Proposition 4]. Moreover, $G F_{c}=X$. Take a point $x \in X$ and neighborhood $A$ of $x$ with compact closure. Since the covering $\left\{W_{j}\right\}_{j \in J}$ is locally finite, then the transporters $G_{A U_{i_{j}}}$ from $A$ to $U_{i_{j}}$ are nonempty for only finitely many $j \in J$. Since the sets $A$ and $U_{i_{j}}$ have compact closure and the action $(G, X)$ is proper, then the transporter $G_{A F_{o}}$ of $A$ to $F_{o}$ has compact closure in $G$. Thus, $F_{c}$ and $F_{o}$ are fundamental sets and by construction $F_{c} \subset F_{o} \subset U$.

(ii) Let $F_{c}$ be a closed fundamental set for the action $(G, X)$ like in (i). Define a relation $\mathscr{R}$ on $F_{c}$ with $x \mathscr{R} y, x, y \in F_{c}$ if and only if $y \in G x$. We will find a Borel section for the closed fundamental set $F_{c}$ with respect to the previous natural relation on $F_{c}$ and then we will show that it is, also, a Borel section for the action $(G, X)$. Obviously $\mathscr{R}$ is an equivalence relation on the separable metric space $\left(F_{c}, d\right)$. Since the action $(G, X)$ is proper each orbit $G x$ is closed in $X$, for every $x \in X$. The $\mathscr{R}$-equivalence class of a point $x \in F_{c}$ is $G x \cap F_{c}$, hence it 
is a closed subset of $X$, thus it is a complete space with respect to the metric $d$. If $U$ is an open subset of $F_{c}$ with respect to the relative topology of $F_{c}$ then the $\mathscr{R}$-saturation of $U$ is the set $G U \cap F_{c}$ which is open in $F_{c}$ hence it is a Borel set. Therefore we can apply [Kallman and Mauldin 1978, Lemma 2] to find a Borel section $S_{B} \subset F_{C}$ for the equivalence relation $\mathscr{R}$. Moreover, $S_{B}$ is a Borel section (and a fundamental set) for the action $(G, X)$, since it is contained in the closed fundamental set $F_{c}$.

Remark 7.5. Note that the assumption that the orbit space $G \backslash X$ is paracompact is automatically satisfied for proper isometric actions. So we can apply Theorem 7.4 in both cases.

Remark 7.6. The statement of Theorem 7.4 cannot be improved by asserting that "There always exists a section $S$ homeomorphic to the orbit space," even if we omit the requirement that a neighborhood $U$ of $S$ is given, as the following simple example shows: Let $\varphi$ be the rotation by $\pi / 2$ on the unit circle and $G$ be the group with two elements generated by $\varphi$. The orbit space is homeomorphic to the halfopen interval $(0,1]$ endowed with a non-Euclidean topology (that is, a sequence tending to 0 converges to 1 ), therefore it cannot be embedded in $S^{1}$.

An answer to the question of whether $S$ can be chosen to be homeomorphic to the orbit space may lead to interesting structure-theorems.

Question 7.7. As Theorem 7.4(ii) indicates the notion of a Borel section is remarkably related to that of a fundamental set in the locally compact case and may be, similarly, used for structural theorems. Note that the Borel section $S_{B}$, because of its construction, is a minimal fundamental set for the action $(G, X)$, that is, for each point $x \in X$ the transporter $G_{\{x\} S_{B}}=g G_{x}$ for some $g \in G$. So, it is interesting to ask whether the existing Borel section for the action $\left(E, X \cup X_{l}\right)$ can be reduced, or lead, to a Borel section for the initial action (Iso $(X), X)$.

\section{References}

[Abels 1972] H. Abels, "Enden von Räumen mit eigentlichen Transformationsgruppen”, Comment. Math. Helv. 47 (1972), 457-473. MR 47 \#5852 Zbl 0253.54039

[Bourbaki 1966a] N. Bourbaki, Elements of mathematics: General topology, vol. 1, Hermann, Paris, 1966. MR 34 \#5044a Zbl 0301.54001

[Bourbaki 1966b] N. Bourbaki, Elements of mathematics: General topology, vol. 2, Hermann, Paris, 1966. MR 34 \#5044b Zbl 0301.54002

[Engelking 1989] R. Engelking, General topology, 2nd ed., Sigma Series in Pure Mathematics 6, Heldermann, Berlin, 1989. MR 91c:54001 Zbl 0684.54001

[Hjorth 2008] G. Hjorth, "An oscillation theorem for groups of isometries", Geom. Funct. Anal. 18:2 (2008), 489-521. MR 2009f:03059 Zbl 1153.03028

[Kallman and Mauldin 1978] R. R. Kallman and R. D. Mauldin, "A cross section theorem and an application to $C^{*}$-algebras", Proceedings Amer. Math. Soc. 69:1 (1978), 57-61. MR 57 \#3342 Zbl 0381.28001 
[Koszul 1965] J.-L. Koszul, Lectures on groups of transformations, Tata Inst. Fund. Res. Studies in Math. 32, Tata Institute of Fundamental Research, Bombay, 1965. MR 36 \#1571 Zbl 0195.04605

[Manoussos and Strantzalos 2007] A. Manoussos and P. Strantzalos, "On embeddings of proper and equicontinuous actions in zero-dimensional compactifications", Trans. Amer. Math. Soc. 359:11 (2007), 5593-5609. MR 2008f:37022 Zbl 1124.37004

Received September 6, 2011.

\author{
ANTONIOS MANOUSSOS \\ FAKULTÄT FÜR MATHEMATIK, SFB 701 \\ UNIVERSITÄT BIELEFELD \\ POSTFACH 100131 \\ D-33501 BIELEFELD \\ GERMANY \\ amanouss@math.uni-bielefeld.de \\ POLYCHRONIS STRANTZALOS \\ DEPARTMENT OF MATHEMATICS \\ UNIVERSITY OF ATHENS \\ PANEPISTIMIOUPOLIS \\ GR-157 84 ATHENS \\ GREECE \\ pstrantz@math.uoa.gr
}




\title{
PACIFIC JOURNAL OF MATHEMATICS
}

\author{
http://pacificmath.org \\ Founded in 1951 by \\ E. F. Beckenbach (1906-1982) and F. Wolf (1904-1989)
}

\section{EDITORS}

V. S. Varadarajan (Managing Editor)

Department of Mathematics

University of California

Los Angeles, CA 90095-1555

pacific@math.ucla.edu

Vyjayanthi Chari

Department of Mathematics

University of California

Riverside, CA 92521-0135

chari@math.ucr.edu

\section{Robert Finn}

Department of Mathematics Stanford University

Stanford, CA 94305-2125

finn@math.stanford.edu

Kefeng Liu

Department of Mathematics

University of California

Los Angeles, CA 90095-1555

liu@math.ucla.edu
Darren Long

Department of Mathematics

University of California

Santa Barbara, CA 93106-3080

long@math.ucsb.edu

Jiang-Hua Lu

Department of Mathematics

The University of Hong Kong

Pokfulam Rd., Hong Kong jhlu@maths.hku.hk

Alexander Merkurjev

Department of Mathematics

University of California

Los Angeles, CA 90095-1555

merkurev@math.ucla.edu
Sorin Popa

Department of Mathematics University of California

Los Angeles, CA 90095-1555 popa@math.ucla.edu

Jie Qing

Department of Mathematics

University of California

Santa Cruz, CA 95064

qing@cats.ucsc.edu

Jonathan Rogawski

Department of Mathematics

University of California

Los Angeles, CA 90095-1555

jonr@math.ucla.edu

\section{PRODUCTION}

pacific@math.berkeley.edu

\section{SUPPORTING INSTITUTIONS}

ACADEMIA SINICA, TAIPEI

CALIFORNIA INST. OF TECHNOLOGY INST. DE MATEMÁTICA PURA E APLICADA KEIO UNIVERSITY

MATH. SCIENCES RESEARCH INSTITUTE NEW MEXICO STATE UNIV.

OREGON STATE UNIV.

\author{
STANFORD UNIVERSITY \\ UNIV. OF BRITISH COLUMBIA \\ UNIV. OF CALIFORNIA, BERKELEY \\ UNIV. OF CALIFORNIA, DAVIS \\ UNIV. OF CALIFORNIA, LOS ANGELES \\ UNIV. OF CALIFORNIA, RIVERSIDE \\ UNIV. OF CALIFORNIA, SAN DIEGO \\ UNIV. OF CALIF., SANTA BARBARA
}

\author{
UNIV. OF CALIF., SANTA CRUZ \\ UNIV. OF MONTANA \\ UNIV. OF OREGON \\ UNIV. OF SOUTHERN CALIFORNIA \\ UNIV. OF UTAH \\ UNIV. OF WASHINGTON \\ WASHINGTON STATE UNIVERSITY
}

These supporting institutions contribute to the cost of publication of this Journal, but they are not owners or publishers and have no responsibility for its contents or policies.

See inside back cover or pacificmath.org for submission instructions.

The subscription price for 2012 is US \$420/year for the electronic version, and \$485/year for print and electronic.

Subscriptions, requests for back issues from the last three years and changes of subscribers address should be sent to Pacific Journal of Mathematics, P.O. Box 4163, Berkeley, CA 94704-0163, U.S.A. Prior back issues are obtainable from Periodicals Service Company, 11 Main Street, Germantown, NY 12526-5635. The Pacific Journal of Mathematics is indexed by Mathematical Reviews, Zentralblatt MATH, PASCAL CNRS Index, Referativnyi Zhurnal, Current Mathematical Publications and the Science Citation Index.

The Pacific Journal of Mathematics (ISSN 0030-8730) at the University of California, c/o Department of Mathematics, 969 Evans Hall, Berkeley, CA 94720-3840, is published monthly except July and August. Periodical rate postage paid at Berkeley, CA 94704, and additional mailing offices. POSTMASTER: send address changes to Pacific Journal of Mathematics, P.O. Box 4163, Berkeley, CA 94704-0163.

PJM peer review and production are managed by EditFLOW ${ }^{\mathrm{TM}}$ from Mathematical Sciences Publishers.

PUBLISHED BY PACIFIC JOURNAL OF MATHEMATICS

at the University of California, Berkeley 94720-3840

A NON-PROFIT CORPORATION

Typeset in LATEX

Copyright $(02012$ by Pacific Journal of Mathematics 


\section{PACIFIC JOURNAL OF MATHEMATICS}

Volume $259 \quad$ No. $2 \quad$ October 2012

Flag subdivisions and $\gamma$-vectors

257

Christos A. ATHANASIAdis

Rays and souls in von Mangoldt planes

279

IGOR BELEGRADEK, ERIC CHOI and NOBUHIRO INNAMI

Isoperimetric surfaces with boundary, II

ABRAHAM FRANDSEN, DONALD SAMPSON and NEIL

STEINBURG

Cyclic branched coverings of knots and quandle homology

YUICHI KABAYA

On a class of semihereditary crossed-product orders

JOHN S. KAUTA

An explicit formula for spherical curves with constant torsion

DEMETRE KAZARAS and IVAN STERLING

Comparing seminorms on homology

JEAN-FranÇOIS LAFONT and CHRISTOPHE PITTET

Relatively maximum volume rigidity in Alexandrov geometry

NAN LI and XIAOCHUN RONG

Properness, Cauchy indivisibility and the Weil completion of a group of 421 isometries

Antonios Manoussos and Polychronis Strantzalos

Theta lifts of strongly positive discrete series: the case of $(\tilde{\mathrm{Sp}}(n), O(V)) 445$

IVAN MATIĆ

Tunnel one, fibered links

MATt RATHBUN

Fusion symmetric spaces and subfactors

HANS WENZL 